\title{
Intervenciones dirigidas a la disminución de los síntomas obsesivo-compulsivos en personas con trastorno del espectro autista: una revisión
}

\section{Interventions aimed at reducing obsessive-compulsive symptoms in people with autism spectrum disorder: a review}

\author{
Maite Montagut-Asunción ${ }^{1}$, Elan Joel Flores-Pérez², \\ Amparo Tijeras-Iborra², Gemma Pastor-Cerezuela ${ }^{2}$ \\ 'Universidad Católica de Valencia, España \\ ${ }^{2}$ Universitat de València, España
}

\begin{abstract}
Autism spectrum disorder (ASD) is a neurodevelopmental disorder characterized by deficits in communication and social interaction as well as restrictive and repetitive behavior. It has high comorbidity with other disorders, reaching $37 \%$ with obsessive-compulsive disorder (OCD) in young patients. OCD is characterized by recurrent thoughts and ideas that lead the person to perform compulsive actions to reduce the level of anxiety generated by these thoughts. This paper presents a review of the psychological intervention methods, as well as their effectiveness, used to reduce the symptoms of comorbid OCD in people with ASD. It includes 14 articles in which cognitive behavioral therapy (CBT) and exposure and prevention of responses (EPR) showed encouraging results in reducing these symptoms.
\end{abstract}

Keywords: autism spectrum disorder, obsessive compulsive disorder, comorbidity, intervention, review.

\begin{abstract}
Resumen
El trastorno del espectro autista (TEA) es un trastorno del neurodesarrollo caracterizado por déficits en la comunicación e interacción social y por comportamientos restrictivos y repetitivos. Presenta una elevada comorbilidad con otros trastornos, de hasta un $37 \%$ con el trastorno obsesivo compulsivo (TOC) en pacientes jóvenes. El TOC se caracteriza por pensamientos e ideas recurrentes que llevan a la persona a realizar acciones compulsivas para disminuir el nivel de ansiedad que estos pensamientos le generan. Este trabajo presenta una revisión de los métodos psicológicos de intervención utilizados, así como de la efectividad de los mismos, en la reducción de los síntomas del TOC comórbido en personas con TEA. Se incluyeron 14 artículos, en los que la terapia cognitivo conductual (TCC) y la exposición y prevención de respuestas (EPR) presentaron resultados alentadores en la disminución de estos síntomas.
\end{abstract}

Palabras Clave: trastorno del espectro autista, trastorno obsesivo compulsivo, comorbilidad, intervención, revisión.

Cómo citar este artículo: Montagut-Asunción, M., Flores-Pérez, E. J., Tijeras-lborra, A., \& Pastor-Cerezuela, G. (2020). Inter-venciones dirigidas a la disminución de los síntomas obsesivo-compulsivos en personas con trastorno del espectro autista: una revisión. Escritos de Psicología - Psychological Writings, 13(2), 80-89.

https://doi.org/1024310/espsiescpsi.v13i2.12414

Correspondencia: Maite Montagut-Asunción. Universidad Católica de Valencia. Facultad de Psicología. Avenida de la llustración, 2. 46100 BURJASOT, Valencia. E-mail: maite.montagut@ucv.es Coautores: Elan Joel Flores-Pérez elan_flores@ hotmail.es, Amparo Tijeras-Iborra m.amparo.tijeras@uv.es, Gemma Pastor-Cerezuela gemma.pastor@uv.es 


\section{Introducción}

El trastorno del espectro autista (TEA) es un trastorno del neurodesarrollo que se caracteriza por la presencia de limitaciones en la comunicación e interacción social en múltiples contextos, así como por comportamientos e intereses restrictivos y repetitivos (DSM-5, APA, 2013). Los posibles niveles de gravedad o severidad del trastorno definidos en el DSM-5 marcan el nivel de apoyo requerido por la persona. De acuerdo con la Organización Mundial de la Salud (OMS, 2018) 1 de cada 160 niños presenta este trastorno.

Por su parte, el trastorno obsesivo-compulsivo (TOC) es un trastorno que se caracteriza por la presencia de pensamientos, ideas o sensaciones recurrentes e indeseables, en ocasiones irracionales, denominadas obsesiones, que llevan a la persona a realizar acciones repetitivas. Los criterios diagnósticos (DSM-5, APA, 2013) incluyen la presencia de obsesiones y/o compulsiones que ocupan más de una hora al día de su tiempo en realizarlas y generan altos niveles de angustia. Estos síntomas interfieren directamente en la vida cotidiana y en el desarrollo social o en otras funciones importantes de la persona. A pesar de ser uno de los trastornos más incapacitantes y afectar al 3\% de la población mundial, solo el 43\% de las personas con este trastorno son atendidas (Gómez et al., 2018).

EI TEA y el TOC son dos trastornos con alto riesgo de comorbilidad. Se calcula que la comobrbilidad del TOC llega a ser del $37 \%$ en casos de jóvenes diagnosticados de TEA (Leyfer et al., 2006) y del $25 \%$ en pacientes adultos con TEA (Cadman et al., 2015; Rydén \& Bejerot, 2008). Partiendo de este hecho, es imperante el hacer un correcto diagnóstico diferencial. En este sentido, una cuestión clave es la correcta diferenciación entre las obsesiones y compulsiones del TOC y los patrones de comportamiento, intereses y actividades repetitivas y estereotipadas del TEA. En el caso del TEA, estas conductas suelen ser egosintónicas, es decir, que "la persona se siente bien consigo misma mientras las realiza, sin que ello le genere conflicto o juicio negativo hacia sí mismo llegando a ser, incluso, fuente de placer y agrado, intrínsecamente motivante, y reforzantes" (Paula-Pérez, 2012, p. 181); mientras que en el caso del TOC, las obsesiones y compulsiones son consideradas egodistónicas, es decir, "percibidas como intrusivas y no queridas por la persona que las padece, no placenteras y con un objetivo muy claro de reducir o eliminar el pensamiento obsesivo amenazante" (Paula-Pérez, 2012, p. 181), además de provocar malestar clínico significativo. En lo que respecta a los intereses restrictivos, en el TEA el interés persistente en determinados temas u objetos genera satisfacción al documentarse o al hablar de los mismos. Sin embargo, en el TOC estos intereses responden a pensamientos intrusivos que, en muchas ocasiones, las personas que los presentan denotan que son irracionales.

Si tenemos en cuenta estos índices de prevalencia del TEA con TOC comórbido y la importancia de un buen diagnóstico diferencial, cobra sentido el conocer los métodos psicológicos de intervención y la eficacia de los mismos, pues son evidentes las repercusiones clínicas de este cuadro y las consecuencias que conlleva en la vida cotidiana de las personas que lo presentan.

El método de intervención psicológica más utilizado para el tratamiento del TOC comórbido con el TEA es la terapia cognitivo-conductual (TCC), un tratamiento psicológico encaminado a modificar los pensamientos, sentimientos y comportamientos negativos y que incluye: 1) La psicoeducación, que és un método terapéutico encaminado a educar al paciente, grupo y familiares acerca del diagnóstico, proporcionándoles información de interés referente al trastorno; 2) El mapeo de síntomas del TOC, técnica que, junto a la psicoeducación, pretende hacer consciente -al paciente, familia y terapeuta- de los síntomas que persisten en el paciente, empleando elementos gráficos donde el paciente pueda señalar el nivel de angustia, ansiedad, estrés o enojo que experimenta al presentarse alguno de estos síntomas en determinadas situaciones; 3) Exposición y prevención de respuestas (EPR), técnica que pretende habituar al sujeto a los estímulos aversivos que provocan determinadas obsesiones o compulsiones a tal grado que la ansiedad aumente progresivamente hasta que, sin emitir ninguna conducta para evitarla, esta misma ansiedad disminuya de manera natural hasta un punto que resulte gestionable (Valaer-Rubio, 2015).

Otro método psicológico de intervención en estos casos es el "anxiety management" (AM), que incluye educación sobre la ansiedad, respiración y práctica diafragmática, educación y práctica para la relajación muscular progresiva, educación sobre el estado de ánimo, hábitos saludables y resolución de problemas. También la terapia cognitivo - conductual basada en funciones (TCC-BF) ha demostrado ser efectiva. Este tipo de intervención parte de las propias habilidades del paciente y se basa en los recursos que este posee (cognitivos y/o conductuales) para contrarrestar los síntomas del TOC. Por último, el análisis conductual aplicado basado en funciones (ACA-BF), basado en el paradigma operante skinneriano, pretende sustituir una conducta por otra más deseable y trata de determinar las contingencias de la respuesta, la motivación para ejecutarla, los estímulos que la anteceden y las consecuencias que la mantienen (Rodas, 2009). 
El objetivo del presente trabajo fue realizar una revisión de los métodos psicológicos de intervención utilizados para el tratamiento del TOC comórbido con el TEA, así como de la efectividad de los mismos.

\section{Método}

Los estudios de la presente revisión se identificaron mediante la búsqueda en el campo de temas de las siguientes bases de datos: Scopus, Web of Science (WOS), PsycINFO y Pub Med, utilizando las palabras claves: intervention, obsessive compulsive disorder, autism spectrum disorder, incluyendo todos los artículos comprendidos en el rango de años 2000-2019 (ambos inclusive) y utilizando la palabra "AND" como operador booleano.

De esta primera búsqueda sistemática, con los descriptores que se especifican más arriba y con la restrincción de años 2000-2019, se obtuvieron 608 artículos: 207 artículos en PubMed, 149 artículos en PsycINFO, 135 artículos en WOS y 117 artículos en Scopus. A partir de estos resultados, se hizo una selección manual con los siguientes criterios de inclusión y exclusión:

Criterios de inclusión:

- Artículos cuya muestra tuviera la categoría diagnóstica de TEA, con la presencia de sintomatología de TOC o de un diagnóstico comórbido de TOC.

- Artículos cuyo objeto de estudio fuera la intervención en la reducción de conductas obsesivo - compulsivas.

- Artículos que incluyeran investigaciones empíricas.

- Artículos en inglés o español.

Criterios de exclusión:

- Artículos cuyo objeto de estudio fuese la intervención farmacológica.

- Artículos que incluyeran solo revisiones teóricas.

- Artículos que no utilizaran una metodología empírica.

- Documentos tipo ensayo, carta, editorial, comentario o publicaciones similares.

\section{Figura 1}

Diagrama de flujo de la selección de los artículos

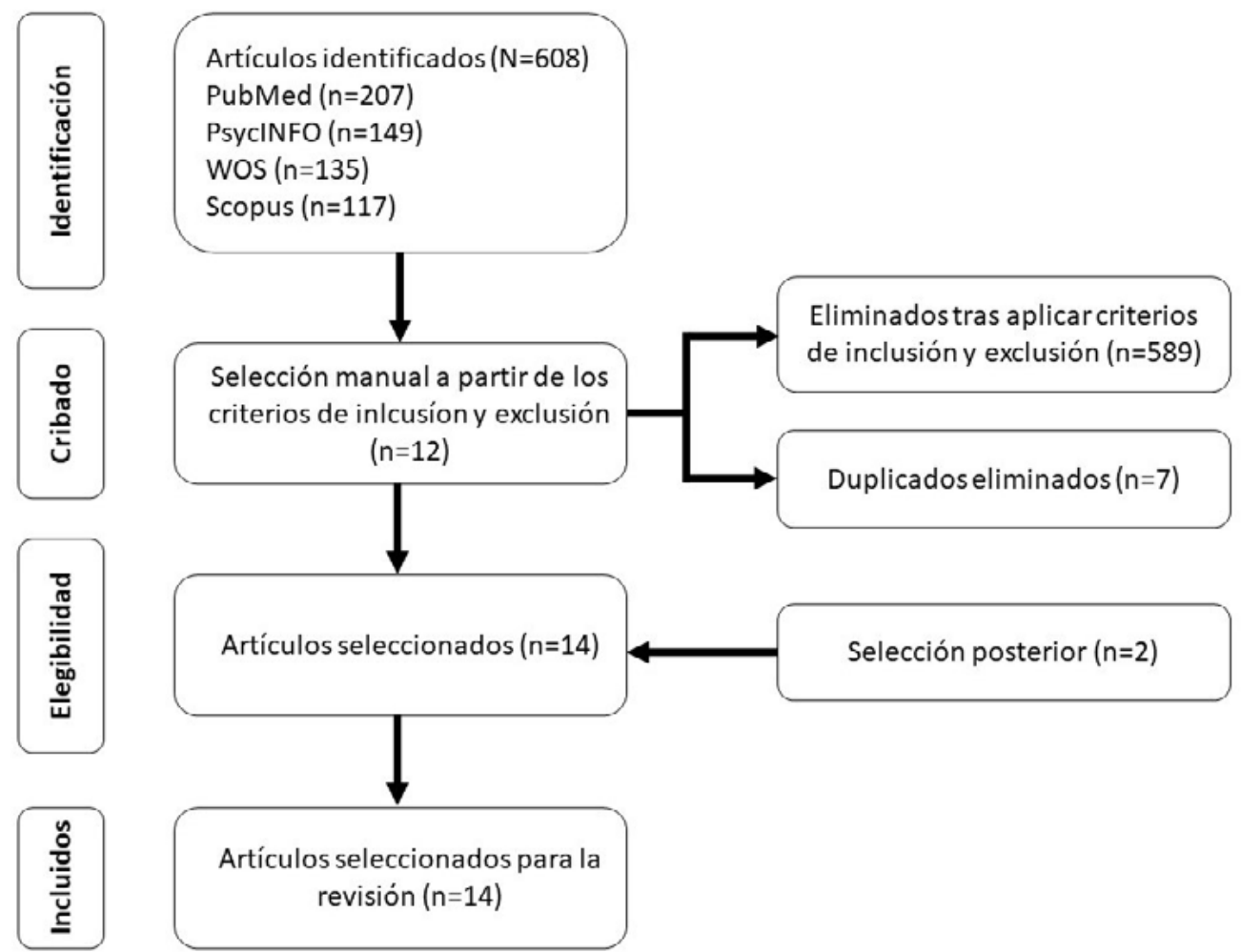

Después de esta selección manual, el número de artículos obtenidos fue de 12 (tras eliminar 7 duplicados). Además, se añadieron a estos 12 artículos, 2 artículos que fueron seleccionados por encontrarse en la bibliografía de otros artículos y porque se consideró que podían aportar una información valuosa en relación con el objetivo del presente trabajo. 
El número final de trabajos que fueron seleccionados para la revisión bibliográfica fue de catorce. El procedimiento de selección de los artículos aparece representado en el diagrama de flujo de la Figura 1.

\section{Resultados}

La información de los 14 artículos revisados se resume en la Tabla 1, en la que se presentan los estudios en orden cronológico, incluyendo: autores y año de publicación, tipo (o tipos) de terapia utilizada en la intervención psicológica, diseño del estudio, síntomas de TOC tratados (en los casos en que se especifican), duración de la intervención (y seguimiento, en los casos en que se especifica), muestra utilizada y resultados obtenidos. Se expone a continuación los principales resultados obtenidos, a través de cuatro categorías: participantes, tipo de intervención, objetivos de la intervención y resultados de la intervención.

\section{Participantes}

En los artículos analizados se intervino en un total de 125 pacientes, de los cuales 88 eran varones (70.4\%) y 37 mujeres $(29.60 \%)$, siendo el de Reaven y Hepburn (2003) el único en el que la intervención se realizó en un paciente femenino. Las edades de los pacientes se situaban entre los 7 y 17 años, exceptuando el estudio de Russell et al. (2013), que incluyó a adolescentes y adultos con edades comprendidas entre 14 y 64 años. La mayoría de los artículos incluyeron estudios de casos (e. g. Elliott \& Fitzsimons, 2014; Farrell, James, Maddox, Griffiths \& White, 2016; Iniesta-Sepúlveda et al., 2018; Lehmkuhl, Storch, Bodfish \& Geffken, 2008; Merricks, Nadeau, Ramos, \& Storch (2017); Nadeau, Arnold, Storch \& Lewin, 2014; Neil, Vause, Jaksic \& Feldman, 2017; Reaven \& Hepburn, 2003; Rombough, 2011; Vause, Hoekstra \& Feldman, 2014).

En lo que respecta al diagnóstico clínico, del total de la muestra, 121 participantes (97.5\%) fueron diagnosticados de TOC; en los restantes (2.5\%), los participantes no presentaban el diagnóstico de TOC, pero sí síntomas graves del mismo (Neil et al., 2017; Vause et al., 2014). En cuanto al diagnóstico de TEA, de acuerdo con los criterios del DSM-IV-TR (APA, 2000), 102 participantes (82.25\%) presentaban un diagnóstico dentro de las categorías generales del espectro autista (trastorno generalizado del desarrollo, TGD): 22 de ellos estaban diagnosticados de síndrome de Asperger (SA), 16 de TEA de alto funcionamiento (TEA-AF), 58 de TEA y 7 de TGD - no especificado (TGD-NE). Los 22 restantes (17.7\%) del total de la muestra fueron los participantes del grupo con diagnóstico de TOC (sin TEA) del estudio realizado por Murray, Jassi, Mataix-Cols, Barrow y Krebs (2015), en el que se comparó entre una muestra diagnosticada de TOC con TEA $(n=22)$ y otra muestra de TOC $\sin$ TEA $(n=22)$, con la finalidad de contrastar los beneficios de la intervención en cada grupo.

Además de TEA y TOC, en algunos estudios se incluyeron también pacientes que presentaban otros trastornos comórbidos. Así, en el trabajo de Nadeau et al. (2014) participaba una niña de 9 años que presentaba, además de TEA y TOC, fobia social y fobia específica; en el de Krebs, Murray y Jassi (2016) participaba un niño de 14 años que presentaba TEA, TOC y dislexia; y en el de Farrell et al. (2016) participaba un paciente de 16 años que presentaba trastorno por déficit de atención con hiperactividad (TDAH), además de TEA-AF y TOC. Por último, en el trabajo de Merricks et al. (2017), se hace el seguimiento de un niño de 12 años con TEA de alto funcionamiento y TOC comórbido, y además TDAH, trastorno de ansiedad generalizada y trastorno de tic, así como depresión e ideaciones suicidas.

Entre los trabajos que documentan el $\mathrm{Cl}$ de su muestra, destaca el realizado por Reaven y Hepburn (2003), en el que la participante presentaba un $\mathrm{Cl}$ de 135-145, mientras que en otros trabajos (Krebs et al., 2016; Lehmkuhl et al., 2008; Nadeau et al., 2014; Neil et al., 2017; Rombough, 2011; Vause et al., 2014, 2017) el Cl de los participantes estaba entre borderline y medio. Por último, en otros estudios (Elliott \& Fitzsimons, 2014; Farrell et al., 2016; Iniesta-Sepúlveda et al., 2018; Murray et al., 2015; Russell et al., 2013) no se documentó el Cl de los participantes.

\section{Tipo de Intervención}

La TCC es el método de intervención principal en los 14 artículos analizados. Solo hubo dos estudios en los que se utilizó, además, otros métodos de intervención con la finalidad de poder compararlos. Así, en el ensayo controlado aleatorizado de Russell et al. (2013) también se utilizó el método de "anxiety management" (AM). Aunque los resultados indicaban la eficacia de ambos tipos de intervención, la TCC mostró tener una ligera ventaja en la disminución de los síntomas del TOC. Por su parte, en el ensayo aleatorizado de Vause et al. (2017) se comparó entre la TCC-BF (junto con psicoeducación, mapeo de síntomas de TOC y EPR) y el ACA-BF. Los resultados mostraron mayor eficacia de la TCC-BF en la reducción de los síntomas del TOC, frente al ACA-BF. 
Tabla 1

Síntesis de los estudios revisados

\begin{tabular}{|c|c|c|c|c|c|c|}
\hline Autores y año & Muestra & $\begin{array}{l}\text { Sintomas de TOC } \\
\text { tratados }\end{array}$ & $\begin{array}{l}\text { Diseño del } \\
\text { estudio }\end{array}$ & Tipo de terapia & $\begin{array}{l}\text { Duración de la } \\
\text { intervención }\end{array}$ & Resultados \\
\hline $\begin{array}{l}\text { Reaven \& } \\
\text { Hepburn (2003) }\end{array}$ & $\begin{array}{l}\text { Niña de } 7 \text { años y } 8 \text { meses. } \\
\text { Dx: SA + TOC } \\
\text { Cl: } 135-145\end{array}$ & Síntomas TOC & $\begin{array}{l}\text { Mixto. } \\
\text { Estudio de } \\
\text { caso. }\end{array}$ & $\mathrm{TCC}+\mathrm{EPR}$ & $\begin{array}{l}14 \text { sesiones en } 14 \\
\text { semanas. }\end{array}$ & Reducción de los síntomas del TOC en un $65 \%$. \\
\hline $\begin{array}{l}\text { Lehmkuhl, } \\
\text { Storch, Bodfish, } \\
\text { \& Geffken } \\
\text { (2008) }\end{array}$ & $\begin{array}{l}\text { Niño de } 12 \text { años. } \\
\text { Dx: TEA-AF + TOC } \\
\text { Cl:92 }\end{array}$ & $\begin{array}{l}\text { Conductas ritualistas } \\
\text { y de evitación }\end{array}$ & $\begin{array}{l}\text { Mixto. } \\
\text { Estudio de } \\
\text { caso. }\end{array}$ & $\mathrm{TCC}+\mathrm{EPR}$ & $\begin{array}{l}10 \text { sesiones de } 50 \text { minutos } \\
\text { en } 16 \text { semanas. } \\
\text { Seguimiento: } 3 \text { meses. }\end{array}$ & $\begin{array}{l}\text { Reducción significativa de los sintomas del TOC } \\
\text { según escalas CY-BOCS (Scahill et al., 1997) y } \\
\text { COIS-R (Piacentini et al., 2007). } \\
\text { Mantenimiento de las ganancias a los } 3 \text { meses. }\end{array}$ \\
\hline $\begin{array}{l}\text { Rombough } \\
\text { (2011) }\end{array}$ & $\begin{array}{l}2 \text { pacientes varones de } 9 \text { y } 7 \text { años. } \\
\text { Dx: TEA-AF } \\
+ \text { sintomas graves de TOC } \\
\text { Cl: } 88\end{array}$ & $\begin{array}{l}\text { Conductas obsesivo - } \\
\text { compulsivas }\end{array}$ & $\begin{array}{l}\text { Mixto. } \\
\text { Estudio de } \\
\text { casos. }\end{array}$ & $\mathrm{TCC}+\mathrm{EPR}$ & $\begin{array}{l}12 \text { sesiones semanales. } \\
\text { Seguimiento: } 8 \text { meses. }\end{array}$ & $\begin{array}{l}\text { En ambos casos, reducción de los síntomas del } \\
\text { TOC de graves a leves, según escala CY-BOCS } \\
\text { (Scahill et al., 1997). }\end{array}$ \\
\hline $\begin{array}{l}\text { Russell et al. } \\
(2013)\end{array}$ & $\begin{array}{l}46 \text { adolescentes y adultos ( } 35 \\
\text { hombres y } 11 \text { mujeres). } \\
\text { DX: TEA +TOC. } \\
\text { Asignados aleatoriamente a dos } \\
\text { grupos: TCC // AM }\end{array}$ & Sintomas TOC & $\begin{array}{l}\text { Cuantitativo. } \\
\text { Ensayo } \\
\text { controlado } \\
\text { aleatorizado. }\end{array}$ & TCC // AM & $\begin{array}{l}20 \text { sesiones. } \\
\text { Seguimiento: } 12 \text { meses. }\end{array}$ & $\begin{array}{l}\text { En ambos grupos, reducción significativa de los } \\
\text { síntomas del TOC, aunque con una ligera ventaja } \\
\text { de la TCC. }\end{array}$ \\
\hline $\begin{array}{l}\text { Elliott \& } \\
\text { Fitzsimons } \\
\text { (2014) }\end{array}$ & $\begin{array}{l}\text { Niño de } 7 \text { años. } \\
\text { Dx: TEA + TOC }\end{array}$ & $\begin{array}{l}\text { Miedo a } \\
\text { contaminarse, } \\
\text { lavarse las manos } \\
\text { hasta hacerse } \\
\text { daño, pensamientos } \\
\text { egodistónicos }\end{array}$ & $\begin{array}{l}\text { Cualitativo. } \\
\text { Estudio de } \\
\text { caso. }\end{array}$ & $\mathrm{TCC}+\mathrm{EPR}$ & $\begin{array}{l}10 \text { sesiones semanales, } 2 \\
\text { sesiones de seguimiento y } \\
\text { dos consultas con los } \\
\text { padres a solas. }\end{array}$ & $\begin{array}{l}\text { Reducción del tiempo y de la frecuencia del } \\
\text { lavado de manos a márgenes aceptables por los } \\
\text { terapeutas. } \\
\text { Mejora en capacidad de externalizar las } \\
\text { obsesiones, habilidad verbal, comprensión y } \\
\text { habilidad paterna de participar plenamente en el } \\
\text { proceso terapéutico. }\end{array}$ \\
\hline $\begin{array}{l}\text { Nadeau, Arnold, } \\
\text { Storch, \& Lewin } \\
\text { (2014) }\end{array}$ & $\begin{array}{l}\text { Niño de } 9 \text { años. } \\
\text { Dx: TOC + TEA + Fobia social y } \\
\text { fobia especifica } \\
\text { Cl: Borderline }\end{array}$ & Sintomas TOC & $\begin{array}{l}\text { Cualitativo. } \\
\text { Estudio de } \\
\text { caso. }\end{array}$ & $\mathrm{TCC}+\mathrm{EPR}$ & $\begin{array}{l}16 \text { sesiones semanales de } \\
\text { entre } 35 \text { y } 70 \text { minutos, en } \\
4 \text { meses. } \\
\text { Seguimiento: } 4 \text { meses. }\end{array}$ & $\begin{array}{l}\text { Disminución significativa de los síntomas de TOC } \\
\text { y fobia social, quedando estos diagnósticos en } \\
\text { remisión. } \\
\text { Disminución de la ansiedad de grave a leve. } \\
\text { En los síntomas autistas: mejora en consciencia } \\
\text { social, comunicación social, motivación social y } \\
\text { manierismos, y ligera reducción en la percepción } \\
\text { de los padres respecto a los déficits de respuesta } \\
\text { social. }\end{array}$ \\
\hline $\begin{array}{l}\text { Vause, Hoekstra, } \\
\& \text { Feldman } \\
\text { (2014) }\end{array}$ & $\begin{array}{l}2 \text { pacientes: } \\
\text { Niño de } 10 \text { años y } 8 \text { meses. } \\
\text { Niña de } 8 \text { años y un mes. } \\
\text { Dx: TEA + TOC } \\
\text { Cl: Borderline }\end{array}$ & $\begin{array}{l}\text { Obsesiones con la } \\
\text { limpieza y conductas } \\
\text { ritualistas }\end{array}$ & $\begin{array}{l}\text { Cuantitativo. } \\
\text { Estudio de } \\
\text { caso. }\end{array}$ & $\begin{array}{l}\text { TCC-BF (EPR + } \\
\text { PsEd. + mapeo) }\end{array}$ & $\begin{array}{l}\text { Niño: } 15 \text { sesiones de } 90 \\
\text { minutos. } \\
\text { Seguimiento: } 3 \text { meses. } \\
\text { Niña: } 11 \text { sesiones de } 60-90 \\
\text { minutos. } \\
\text { Seguimiento: } 4 \text { meses. }\end{array}$ & $\begin{array}{l}\text { Niño: Disminución de los síntomas del TOC de } \\
\text { graves a leves. } \\
\text { Niña: Disminución de los síntomas del TOC de } \\
\text { graves a subclínicos. } \\
\text { Según escala CY-BOCS (Scahill et at., 1997). }\end{array}$ \\
\hline $\begin{array}{l}\text { Murray, Jassi, } \\
\text { Mataix-Cols, } \\
\text { Barrow, \& Krebs } \\
\text { (2015) }\end{array}$ & $\begin{array}{l}44 \text { pacientes en dos grupos: } \\
22 \text { Dx: TEA + TOC ( } 15 \text { SA, } \\
2 \text { TEA-AF y } \\
5 \text { TGD-NE) } \\
22 \text { Dx: TOC }\end{array}$ & Sintomas TOC & $\begin{array}{l}\text { Cuantitativo. } \\
\text { Ensayo } \\
\text { controlado. }\end{array}$ & $\mathrm{TCC}+\mathrm{EPR}$ & $\begin{array}{l}14 \text { sesiones de una hora en } \\
17 \text { semanas. }\end{array}$ & $\begin{array}{l}\text { En ambos grupos, reducción significativa de } \\
\text { los síntomas del TOC, aunque con ganancias } \\
\text { significativamente mayores en el grupo con Dx } \\
\text { TOC (sin TEA). }\end{array}$ \\
\hline $\begin{array}{l}\text { Krebs, Murray, \& } \\
\text { Jassi (2016) }\end{array}$ & $\begin{array}{l}\text { Niño de } 14 \text { años. } \\
\text { Dx: TEA + TOC + Dislexia }\end{array}$ & $\begin{array}{l}\text { Conductas ritualistas } \\
\text { y de evasión }\end{array}$ & $\begin{array}{l}\text { Cuantitativo. } \\
\text { Estudio de } \\
\text { caso. }\end{array}$ & $\mathrm{TCC}+\mathrm{EPR}$ & $\begin{array}{l}18 \text { sesiones en } 4 \text { meses. } \\
\text { Post-test a los } 6 \text { y } 12 \\
\text { meses. }\end{array}$ & $\begin{array}{l}\text { Reducción del } 50 \% \text { en los sintomas del TOC. } \\
\text { Mantenimiento de las ganancias a los } 12 \text { meses. }\end{array}$ \\
\hline $\begin{array}{l}\text { Farrell, James, } \\
\text { Maddox, } \\
\text { Griffiths, \& White } \\
\text { (2016) }\end{array}$ & $\begin{array}{l}\text { Chico de } 16 \text { años. Dx: TEA-AF + } \\
\text { TOC + TDAH }\end{array}$ & $\begin{array}{l}\text { Rituales de ducharse, } \\
\text { limpieza e ir al baño }\end{array}$ & $\begin{array}{l}\text { Cuantitativo. } \\
\text { Estudio de } \\
\text { caso. }\end{array}$ & $\begin{array}{l}\text { TCC + EPR + } \\
\text { PsEd. }\end{array}$ & $\begin{array}{l}3 \text { sesiones de } 3 \text { horas + } \\
1 \text { sesión de PsEd. a la } \\
\text { familia. } \\
\text { Seguimiento: } 3 \text { sesiones } \\
\text { de } 1 \text { hora por video } \\
\text { conferencia, por } 3 \text { meses. }\end{array}$ & $\begin{array}{l}\text { Después de } 3 \text { meses del tratamiento, remisión } \\
\text { completa del TOC. } \\
\text { Reducción sistemática de la angustia provocada } \\
\text { por las conductas ritualistas. }\end{array}$ \\
\hline $\begin{array}{l}\text { Vause, } \\
\text { Neil, Jaksic } \\
\text { Jackiewicz, \& } \\
\text { Feldman (2017) }\end{array}$ & $\begin{array}{l}14 \text { pacientes } \\
\text { (9 niños y } 5 \text { niñas) de } 8 \text { a } 12 \text { años. } \\
\text { Dx: TEA+ TOC ( } 6 \text { Autismo, } 6 \text { SA y } \\
2 \text { TGD-NE). } \\
\text { Asignados aleatoriamente a dos } \\
\text { grupos: } \\
\text { TCC-BF // ACA-BF } \\
\text { Cl } \geq 70\end{array}$ & $\begin{array}{l}\text { Conductas } \\
\text { compulsivas, } \\
\text { ritualistas y de } \\
\text { necesidad de } \\
\text { invarianza }\end{array}$ & $\begin{array}{l}\text { Cuantitativo. } \\
\text { Ensayo } \\
\text { controlado } \\
\text { aleatorizado. }\end{array}$ & $\begin{array}{l}\text { TCC-BF (PsEd. } \\
+ \text { mapeo + EPR) } \\
\text { // ACA-BF }\end{array}$ & $\begin{array}{l}2 \text { horas semanales en } \\
5 \text { meses. } \\
\text { Seguimiento: } 5 \text { meses. }\end{array}$ & $\begin{array}{l}\text { Reducción significativa en las conductas } \\
\text { compulsivas, } \\
\text { ritualistas y de necesidad de invarianza solo en } \\
\text { el grupo } \\
\text { TCC-BF. }\end{array}$ \\
\hline $\begin{array}{l}\text { Neil, Vause, } \\
\text { Jaksic, \& } \\
\text { Feldman (2017) }\end{array}$ & $\begin{array}{l}\text { Niño de } 11 \text { años. } \\
\text { Dx. TEA+ sintomas TOC } \\
\text { Cl: Borderline }\end{array}$ & $\begin{array}{l}\text { Rasgar papel, oler } \\
\text { papel y contar dedos } \\
\text { en orden específico }\end{array}$ & $\begin{array}{l}\text { Cuantitativo. } \\
\text { Estudio de } \\
\text { caso. }\end{array}$ & $\begin{array}{l}\text { TCC-BF + PsEd. } \\
\text { + mapeo + EPR }\end{array}$ & $\begin{array}{l}9 \text { sesiones de } 2 \text { horas } \\
\text { semanales. } \\
\text { Seguimiento: } 6,12 \text { y } 20 \\
\text { meses. }\end{array}$ & $\begin{array}{l}\text { Reducción considerable en las conductas de } \\
\text { rasgar papel, oler papel y contar dedos en orden } \\
\text { específico. } \\
\text { Aumento en habilidades psicosociales. } \\
\text { Alta satisfacción del paciente y la familia. }\end{array}$ \\
\hline $\begin{array}{l}\text { Merricks, } \\
\text { Nadeau, Ramos, } \\
\text { \& Storch (2017) }\end{array}$ & $\begin{array}{l}\text { Niño de } 12 \text { años. } \\
\text { Dx. TEA + TOC comórbido + } \\
\text { TDAH + Trastorno de ansiedad } \\
\text { generalizada y trastorno de tic } \\
\text { TEA de alto funcionamiento }\end{array}$ & $\begin{array}{l}\text { Rituales de abrir y } \\
\text { cerrar elementos, } \\
\text { respiración profunda, } \\
\text { abrir y cerrar el } \\
\text { agua, rezar y / o } \\
\text { pedir perdón a Dios } \\
\text { constantemente. }\end{array}$ & $\begin{array}{l}\text { Cuantitativo. } \\
\text { Estudio de } \\
\text { caso. }\end{array}$ & $\mathrm{TCC}+\mathrm{EPR}$ & $\begin{array}{l}33 \text { sesiones durante } 7 \\
\text { semanas. } \\
5 \text { dias a la semana }(6,5 \\
\text { horas al dia) con un familiar. }\end{array}$ & $\begin{array}{l}\text { La puntuación en el CY-BOCS, que inicialmente } \\
\text { era "Extrema", con una puntuación de } 38 \text { (de } 40 \text { ), } \\
\text { disminuyó significativamente a una puntuación } \\
\text { subclínica de } 4 \text {. } \\
\text { La puntuación acumulada en ansiedad infantil } \\
\text { (Screen for Child Anxiety Related Disorders; } \\
\text { Birmaher, Khetarpal, Cully, } \\
\text { Brent, \& McKenzie, 1995), que inicialmente era de } \\
\text { 39, se redujo a cero. }\end{array}$ \\
\hline $\begin{array}{l}\text { Iniesta- } \\
\text { Sepúlveda et al. } \\
\text { (2018) }\end{array}$ & $\begin{array}{l}9 \text { pacientes ( } 8 \text { chicos y } 1 \text { chica), } \\
\text { de } 11 \text { a } 17 \text { años. } \\
\text { Dx. TEA -AF + TOC }\end{array}$ & $\begin{array}{l}\text { Conductas obsesivas } \\
\text { y compulsivas }\end{array}$ & $\begin{array}{l}\text { Cuantitativo. } \\
\text { Estudio de } \\
\text { casos. }\end{array}$ & $\mathrm{TCC}+\mathrm{EPR}$ & $\begin{array}{l}3 \text { horas diarias, } 5 \text { dias } \\
\text { semanales, en } 24 \text { - } 80 \\
\text { dias, dependiendo de cada } \\
\text { paciente. }\end{array}$ & $\begin{array}{l}\text { Reducción significativa de los síntomas del TOC en } \\
\text { el } 76 \% \text { de los pacientes. } \\
\text { Mejoras en la calidad de vida social. } \\
\text { Reducción de los índices de ansiedad y depresión. }\end{array}$ \\
\hline
\end{tabular}

Nota. TCC $=$ Terapia cognitivo conductual. EPR = exposición y prevención de respuestas. $A M=$ anxiety management. PsEd. = Psicoeducación. TCC-BF = terapia cognitivo conductual basada en funciones. ACA-BF= análisis conductual aplicado basado en funciones. $\mathrm{Dx}=$ Diagnóstico clínico. $\mathrm{SA}=$ síndrome de Asperger. $\mathrm{TOC}=$ trastorno obsesivo compulsivo. TEA= trastorno de espectro autista. TEA-AF: trastorno de espectro autista de alto funcionamiento. TGD-NE: trastorno generalizado del desarrollo no especificado. TDAH: trastorno por deficit de atención con hiperactividad. $\mathrm{Cl}=\mathrm{cociente}$ intelectual. CY-BOCS= Escala Obsesivo-Compulsiva para Niños de Yale-Brown (Child Yale-Brown Obsessive-Compulsive Scale). COIS-R= Escala del Impacto Obsesivo Compulsivo para Niños-Revisada (Child Obsessive-Compulsive Impact Scale-Revised). 
En otros estudios (Farrell et al., 2016; Iniesta-Sepúlveda et al., 2018; Murray et al., 2015; Neil et al., 2017; Vause et al., 2014, 2017) también se utilizó la psicoeducación dentro de la intervención realizada y en dos de ellos se realizó, además, entrenamiento específico para padres (Neil et al., 2017; Vause et al., 2017). No obstante, en todos estos estudios los padres participaron en la intervención. Un denominador común en todos los trabajos fue que la EPR se implementó en todas las intervenciones. Del total de estudios analizados, solo cuatro documentaron haber tenido participantes sometidos a tratamientos farmacológicos junto con la intervención psicológica (Iniesta-Sepúlveda et al., 2018; Merricks et al. (2017); Reaven \& Hepburn, 2003; Vause et al., 2017).

El número de sesiones de intervención varió entre las 7 sesiones (Lehmkuhl et al., 2008) y 46.5 (media de sesiones en Iniesta-Sepúlveda et al., 2018), desde un intervalo de 9 semanas (Neil et al., 2017) hasta 21 semanas (Nadeau et al., 2014), mientras que la duración de las sesiones osciló entre los 35 minutos (Nadeau et al., 2014) y las 3 horas (Iniesta-Sepúlveda et al., 2018).

\section{Objetivos de la intervención}

Todas las intervenciones fueron encaminadas a tratar síntomas específicos o generales del TOC. De los catorce artículos analizados, solo ocho especificaron en qué conductas en particular centraron su intervención. Así, Lehmkuhl et al. (2008) centraron la intervención en la reducción de conductas ritualistas y de evasión; Farrell et al. (2016) diseñaron su intervención en torno a las rutinas de ducharse, ir al baño y de limpieza que presentaba su paciente. Elliott y Fitzsimons (2014), por su parte, trataron los miedos irracionales hacia la contaminación, el lavado excesivo de manos hasta hacerse daño y los pensamientos egodistónicos presentes en el participante. Vause et al. (2014) delimitaron la intervención a las obsesiones con la limpieza y las conductas ritualistas predominantes en el sujeto. Vause et al. (2017) trabajaron en torno a los comportamientos compulsivos ritualistas y de necesidad de invarianza. Krebs et al. (2016) intervinieron en las conductas ritualistas y de evasión. Neil et al. (2017) centraron su intervención en tres conductas específicas: cortar papel, oler papel rasgado y contar los dedos en estricto orden. Por último, Merricks et al. (2017) centraron la intervención en reducir rituales como abrir y cerrar elementos, respiración profunda, abrir y cerrar el agua, rezar y / o pedir perdón a Dios constantemente.

En los seis estudios restantes (Iniesta-Sepúlveda et al., 2018; Murray et al., 2015; Nadeau et al., 2014; Reaven \& Hepburn, 2003; Rombough, 2011; Russell et al., 2013) la intervención no se llevó a cabo en torno a conductas específicas de los participantes, sino que, de manera más general, el objetivo fue reducir los síntomas del TOC.

\section{Resultados de la Intervención}

Para evaluar la eficacia de las intervenciones se utilizaron varios instrumentos psicométricos tanto al inicio como al final de las mismas. La Escala Obsesivo-Compulsiva para Niños de Yale-Brown (Child Yale-Brown Obsessive-Compulsive Scale CY-BOCS, Scahill et al., 1997) fue la más utilizada para evaluar la disminución de los síntomas del TOC. Esta escala fue utilizada en todos los estudios, excepto el de Elliott y Fitzsimons (2014). Otra escala referida por cuatro de los estudios analizados (Lehmkuhl et al., 2008; Neil et al., 2017; Rombough, 2011; Vause et al., 2017) fue la Escala del Impacto Obsesivo Compulsivo para Niños - Revisada (Child Obsessive-Compulsive Impact Scale-Revised COIS-R, Piacentini, Peris, Bergm, Chang \& Jaffer, 2007). En tres de ellos también se utilizó la Escala de Impresión Clínica Global - Intensidad (Clinical Global Impression-Severity CGI-S, National Institute of Mental Health, 1985). En dos de ellos además se utilizó la Escala de Acomodación Familiar (Family Accommodation Scale FAS, Calvocoressi et at., 1995), la Escala de Comportamiento Repetitivo - Revisada (The Repetitive Behavior Scale-Revised RBS-R, Bodfish, Simons \& Lewis, 1999) y el Inventario Obsesivo Compulsivo para Niños (The Children's Obsessive-Compulsive Inventory ChOCl, Shafran et at., 2003). El único trabajo en el que no se utilizó ninguna herramienta psicométrica para evaluar a sus pacientes fue el de Elliott y Fitzsimons (2014), en el que se realizó entrevista al inicio y al final de la intervención.

El seguimiento de los tratamientos abarcó desde 2 sesiones (Elliott \& Fitzsimons, 2014) hasta 20 meses (Neil et al., 2017). En algunos trabajos (Iniesta-Sepúlveda, et al., 2018; Murray, et al., 2015) solo llevaron a cabo post-test al finalizar el plan de intervención. Todos los resultados denotaron haber tenido efectos positivos respecto a la reducción de los síntomas del TOC, manteniendo los beneficios en el periodo posterior de seguimiento. En cinco de los estudios esta reducción implicó la práctica eliminación del diagnóstico de TOC o de los indicadores subclínicos del mismo (Farrell et al., 2016; Lehmkuhl et al., 2008; Merricks et al., 2017; Nadeau et al., 2014; Vause et al., 2014). En algunos casos, esta reducción fue del 50-65\% de los síntomas (Krebs et al., 2016; Reaven \& Hepburn, 2003). En otros casos, los autores refirieron la reducción de los síntomas del TOC como significativa (Iniesta-Sepúlveda 
et al., 2018; Murray et al., 2015; Russell et al., 2013; Vause et al., 2017). En el caso de Rombough (2011), los síntomas pasaron de ser graves a leves. En algún otro trabajo (Krebs et al., 2017), en el que se abordaban conductas obsesivo-compulsivas concretas, éstas se redujeron a niveles aceptables para los terapeutas.

Además de las reducciones en la sintomatología del TOC, se presentaron otros beneficios adicionales a partir del tratamiento. Así, en el estudio de Farrell et al., (2016) se produjo una reducción sistemática de la angustia provocada por las conductas ritualistas. En el de Elliott y Fitzsimons (2014) el tratamiento tuvo repercusiones positivas en el paciente, en concreto en la capacidad de externalizar las obsesiones, la habilidad verbal, la comprensión paterna y la habilidad paterna de participar plenamente en el proceso terapéutico. En el estudio de Nadeau et al., (2014) se produjo una disminución de la ansiedad de grave a leve en el paciente y, respecto a los síntomas autistas, una mejora en la consciencia social, la comunicación social, la motivación social, una reducción de los manierismos y una ligera reducción en la percepción de los padres de los déficits de respuesta social. En el estudio de Iniesta-Sepúlveda et al., (2018), entre los beneficios de la intervención, también se produjo un aumento en la calidad de vida social y disminución de la ansiedad y depresión del paciente. En el de Neil et al., (2017) se produjo un alto nivel de satisfacción de los padres posteriormente a la intervención y, por último, en el de Merricks et al. (2017), se reporta una disminución simultánea de las compulsiones y la ideación suicida del paciente.

\section{Discusión}

En la presente revisión, los resultados obtenidos denotan que la intervención más utilizada para rehabilitar la sintomatología de TOC en pacientes diagnosticados de TEA fue la TCC incluyendo la EPR como componente principal, mostrando dicha terapia resultados positivos. Además, los estudios en los que se realizó un seguimiento tras los resultados mostraron perdurabilidad de los logros o mejoría alcanzada, lo que nos permite afirmar que este tipo de intervención es una opción viable para disminuir los síntomas a largo plazo en un corto tiempo de intervención. Parte del éxito en la permanencia de los logros podría deberse a que en todas ellas se involucró a los padres o tutores en el proceso terapéutico, con el fin de que continuaran con las técnicas utilizadas con los pacientes durante las sesiones, permitiendo así mantener los resultados positivos en los mismos.

No obstante, aunque dicha terapia muestra resultados positivos en la disminución de los síntomas del TOC en muestras de sujetos diagnosticados con TEA, los resultados no pueden llegar a ser concluyentes debido a la escasez de estudios y el bajo número de sujetos participantes. Así, aunque 70 (97\%) de los participantes diagnosticados de TEA con sintomatología de TOC (o TOC comórbido) respondieron positivamente al tratamiento, se trata de un bajo número de participantes que limita la posibilidad de extraer conclusiones respecto a la efectividad de dicha intervención. La mayor parte de los artículos analizados eran estudios de casos (a excepción de Iniesta-Sepúlveda et al., 2018; Murray et al., 2015; Russell et al., 2013; Vause et al., 2017). Además, cuatro estudios (Iniesta Sepúlveda et al., 2018; Merricks et al., 2017; Reaven \& Hepburn, 2003; Vause et al., 2017) incluyeron pacientes bajo un tratamiento combinando fármacos-TCC, no pudiendo garantizar en tal caso que la mejoría se deba exclusivamente a la intervención terapéutica realizada.

Una de las limitaciones que encontramos es que los pacientes de las muestras contaban con un grado de autismo funcional (el $\mathrm{Cl}$ de los participantes era igual o superior a 70 en los casos documentados), lo que deja fuera a las personas que se encuentran en el extremo inferior del espectro autista (nivel 3 del DSM-5) y con bajo nivel de capacidad intelectual. Esto no permite, por tanto, generalizar los resultados para poder afirmar que este tipo de intervención sea igual de efectiva ante una sintomatología autística más acentuada y con mayores limitaciones intelectuales. Asimismo, las edades de los participantes (a excepción de Russell et al., 2013), comprendían un rango de entre 7 y 17 años, lo cual excluye a pacientes adultos, delimitando el éxito de este tipo de intervención a pacientes jóvenes, pues no existe evidencia empírica de que tenga los mismos resultados en pacientes adultos.

Aunque el objetivo de las intervenciones era la reducción de la sintomatología de TOC, son pocos los estudios que valoran, además, beneficios o mejoría respecto a la condición del espectro autista u otras categorías diagnósticas presentes en el paciente (Elliott \& Fitzsimons, 2014; Iniesta-Sepúlveda et al., 2018; Nadeau et al., 2014). De esta manera, aunque estos resultados pueden ser alentadores para responder a las necesidades de personas con TEA y TOC comórbido, es necesario continuar investigando sobre los efectos de la intervención incluyendo a pacientes de mayor edad y/o con habilidades comunicativas e intelectuales más limitadas, así como valorar el alcance de los métodos de intervención más allá de la reducción de los síntomas presentados. Asimismo, y aunque el objetivo de este estudio era una revisión teórica desde una perspectiva predominantemente cualitativa, la realización de 
esta misma revisión a nivel cuantitativo y en forma de metaanálisis para futuros trabajos aportaría una información que podría ser de gran ayuda a la hora de considerar los distintos tratamientos en caso de comorbilidad de TOC con TEA.

\section{Conclusiones}

El método más utilizado para tratar los síntomas de TOC en personas con TEA es la TCC con enfoque EPR. Aunque son pocos los estudios controlados aleatorizados realizados, los resultados obtenidos expresan alentadores beneficios en la disminución los síntomas del TOC en personas jóvenes diagnosticadas de TEA, siendo esta la mejor opción para mitigar este cuadro sintomatológico. Cabe destacar que la participación de los padres en el proceso terapéutico es imperante para la mejora de los pacientes. El seguimiento de estos en las tareas cotidianas enmarca en gran medida el éxito obtenido a largo plazo de estas intervenciones. No obstante, aunque estos resultados pueden ser alentadores para responder a las necesidades de personas con TEA y TOC comórbido, estas intervenciones dejan fuera a las personas con TEA que requieren de apoyos muy substanciales (nivel 3 del DSM-5), lo que abre la necesidad de continuar investigando sobre los métodos de intervención en personas con TEA que presentan habilidades comunicativas e intelectuales más limitadas, pues no parece haber evidencia empírica que marque una directriz en la forma de atender a estas personas.

\section{Conflicto de intereses}

No existe conflicto de intereses en el contenido del artículo.

\section{Financiación}

Financiado por el Ministerio de Ciencia, Innovación y Universidades a través del proyecto EDU-201678867R (AEI, FEDER, UE).

\section{Referencias}

1. American Psychiatric Association. (2013). Diagnostic and statistical manual of mental disorders (5th ed.). Arlington: American Psychiatric Publishing.

2. Birmaher, B., Khetarpal, S., Cully, M., Brent, D., \& McKenzie, S. (1995). Screen for Child Anxiety Related Disorders (SCARED). Pittsburgh, PA: Western Psychiatric Institute and Clinic, University of Pittsburgh.

3. Bodfish, J., Symons, F., \& Lewis, M. (1999). The repetitive behavior scale: test manual. Morganton, NC.: Western Carolina Center Research Reports.

4. Cadman, T., Spain, D., Johnston, P., Russell, A., Mataix-Cols, D., Craig, M., ... \& Wilson, C. E. (2015). Obsessive-compulsive disorder in adults with high-functioning autism spectrum disorder: what does self-report with the OCI-R tell us? Autism Research, 8, 477-485. https://doi. org/10.1002/aur.1461

5. Calvocoressi, L., Lewis, B., Harris, B., Trufan, B. S., Goodman, W. K., McDougle, C. J., \& Price, L. H. (1995). Family accommodation in obsessive-compulsive disorder. American Journal of Psychiatry, 152, 441-443. https://doi.org/10.1176/ajp.152.3.441

6. $\quad{ }^{*}$ Elliott, S., \& Fitzsimons, L. (2014). Modified CBT for treatment of OCD in a 7-year-old boy with ASD-a case report. Journal of Child and Adolescent Psychiatric Nursing, 27, 156-159. https:// doi.org/10.1111/jcap.12081

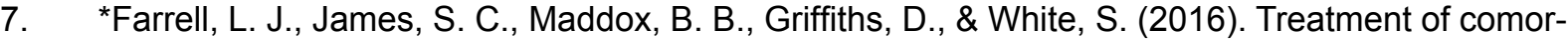
bid obsessive-compulsive disorder in youth with ASD: the case of Max. En E. Storch, \& A. Lewin (Eds.) Clinical Handbook of Obsessive-Compulsive and Related Disorders (pp. 337-355). Switzerland: Springer International Publishing. https://doi.org/10.1007/978-3-319-17139-5 23

8. Gómez, A., Cano, P., Lucas, M., Romero, P., Tolosa, M. \& Martínez, A. (2018). El trastorno obsesivo compulsivo en la infancia y adolescencia. En P. Moreno (Presidencia), Congreso Virtual Internacional de Psiquiatría, Psicología y Salud Mental (Interpsiquis). Conferencia impartida en el XIX Congreso Virtual de Psiquiatría.

9. *IniestaSepúlveda, M., Nadeau, J., Ramos, A., Kay, B., Riemann, B., \& Storch, E. (2018). An initial case series of intensive cognitive-behavioral therapy for obsessive-compulsive disorder in adolescents with autism spectrum disorder. Child Psychiatry \& Human Development, 49, 9-19. https://doi.org/10.1007/s1057

10. ${ }^{*}$ Krebs, G., Murray, K., \& Jassi, A. (2016). Modified cognitive behavior therapy for severe, treatment-resistant obsessive-compulsive disorder in an adolescent with autism spectrum disorder. Journal of Clinical Psychology, 72, 1162-1173. https://doi.org/10.1002/jclp.22396 
11. *Lehmkuhl, H. D., Storch, E. A., Bodfish, J. W., \& Geffken, G. R. (2008). Brief report: exposure and response prevention for obsessive compulsive disorder in a 12-year-old with autism. Journal of Autism and Developmental Disorders, 38, 977-981. https://doi.org/10.1007/s10803-007-0457-2

12. Leyfer, O. T., Folstein, S. E., Bacalman, S., Davis, N. O., Dinh, E., Morgan, J., ... \& Lainhart, J. E. (2006). Comorbid psychiatric disorders in children with autism: interview development and rates of disorders. Journal of Autism and Developmental Disorders, 36, 849-861. https://doi. org/10.1007/s10803-006-0123-0

13. "Merricks, K. L., Nadeau, J. M., Ramos, A., \& Storch, E. A. (2017). A case report of intensive exposure-based cognitive behavioral therapy for a child with comorbid autism spectrum disorder and obsessive-compulsive disorder. Journal of Cognitive Psychotherapy, 31(2), 118-123.

14. "Murray, K., Jassi, A., Mataix-Cols, D., Barrow, F., \& Krebs, G. (2015). Outcomes of cognitive behaviour therapy for obsessive-compulsive disorder in young people with and without autism spectrum disorders: a case controlled study. Psychiatry Research, 228, 8-13. https://doi. org/10.1016/j.psychres.2015.03.012

15. *Nadeau, J., Arnold, E., Storch, E., \& Lewin, A. (2014). Family cognitive-behavioral treatment for a child with autism and comorbid obsessive compulsive disorder. Clinical Case Studies, 13, 22-36. https://doi.org/10.1177/1534650113504488

16. National Institute of Mental Health. (1985). Special feature: rating scales and assessment instruments for use in pediatric psychopharmacology research. Pharmacology Bulletin, 21, 839-843.

17. *Neil, N., Vause, T., Jaksic, H., \& Feldman, M. (2017). Effects of group functional behavior-based cognitive-behavioral therapy for obsessive-compulsive behavior in a youth with autism spectrum disorder. Child \& Family Behavior Therapy, 3, 179-190. https://doi.org/10.1080/07317107.2017. 1338448

18. Organización Mundial de la Salud (2018). Trastornos del espectro autista. Recuperado de https:// www.who.int/es/news-room/fact-sheets/detail/autism-spectrum-disorders

19. Paula-Pérez, I. (2012). Diagnóstico diferencial entre el trastorno obsesivo compulsivo y los patrones de comportamiento, actividades e intereses restringidos y repetitivos en los trastornos del espectro autista. Revista de Psiquiatría y Salud Mental, 6, 178-186. https://doi.org/10.1016/ irpsm.2012.07.005

20. Piacentini, J., Peris, T., Bergm, R., Chang, S., \& Jaffer, M. (2007). Brief report: functional impairment in childhood OCD: development and psychometrics properties of the child obsessive-compulsive impact scale-revised (COIS-R). Journal of Clinical Child \& Adolescent Psychology, 36, 645-653. https://doi.org/10.1080/15374410701662790

21. ${ }^{*}$ Reaven, J., \& Hepburn, S. (2003). Cognitive-behavioral treatment of obsessive compulsive disorder in a child with Asperger syndrome: a case report. Autism, 7, 145-164. https://doi.org/10.11 77/1362361303007002003

22. Rodas, R. (2009). Seguimiento del análisis conductual. En Análisis conductual aplicado (pp. 13-27). Colombia: Universidad de Manizales.

23. *Rombough, E. (2011). Dyad function-based cognitive behavioural therapy as a treatment for obsessive compulsive disorder in two school aged children with high functioning autism. (Unpublished master's thesis) St.Catharines Ontario: Centre of Applied Disability Studies, Brock University. http://hdl.handle.net/10464/3947

24. ${ }^{*}$ Russell, A. J., Jassi, A., Fullana, M. A., Mack, H., Johnston, K., Heyman, I., ... \& Mataix-Cols, D. (2013). Cognitive behavior therapy for comorbid obsessive-compulsive disorder in high-functioning autism spectrum disorders: a randomized controlled trial. Depression and Anxiety, 30, 697-708. https://doi.org/10.1002/da.22053

25. Rydén, E., \& Bejerot, S. (2008). Autism spectrum disorders in an adult psychiatric population: a naturalistic cross-sectional controlled study. Clinical Neuropsychiatry, 5, 13-21.

26. Scahill, L., Riddle, M. A., McSwiggin-Hardin, M., Ort, S. I., King, R. A., Goodman, W. K., ... \& Leckman, J. F. (1997). Children's Yale-Brown obsessive compulsive scale: reliability and validity. Journal of the American Academy of Child \& Adolescent Psychiatry, 36, 844-852. https://doi. org/10.1097/00004583-199706000-00023

27. Shafran, R., Frampton, I., Heyman, I., Reynolds, M., Teachman, B., \& Rachman, S. (2003). Preliminary development of a new self-report measure for OCD in young people. Journal of Adolescence, 26, 137-142. https://doi.org/10.1016/S0140-1971(02)00083-0

28. Valaer-Rubio, Á. (2015). Abordaje clínico y tratamiento del trastorno obsesivo-compulsivo. SeviIla: Publicia. 
29. *Vause, T., Hoekstra, S., \& Feldman, M. (2014). Evaluation of individual function-based cognitive-behavioural therapy for obsessive compulsive behaviour in children with autism spectrum disorder. Journal on Developmental Disabilities, 20, 30-41.

30. *Vause, T., Neil, N., Jaksic, H., Jackiewicz, G., \& Feldman, M. (2017). Preliminary randomized trial of function-based cognitive-behavioral therapy to treat obsessive compulsive behavior in children with autism spectrum disorder. Focus on Autism and Other Developmental Disabilities, 32, 218-228. https://doi.org/1177/1088357615588517

Nota: Las referencias marcadas con un asterisco $\left(^{*}\right)$ contienen los estudios incluidos en la revisión.

RECIBIDO: 25 de abril de 2020 MODIFICADO: 11 de enero de 2021

ACEPTDO: 6 de febrero de 2021 